\title{
DISLOCATION EMISSION AT FIBERS-II. EXPERIMENTS AND MICROSTRUCTURE OF THERMAL PUNCHING
}

\author{
D. C. DUNAND and A. MORTENSEN \\ Department of Materials Science and Engineering, Massachusetts Institute of Technology, \\ Cambridge, MA 02139, U.S.A.
}

(Received 22 October 1990; in revised form 28 January 1991)

\begin{abstract}
A decoration technique is used to observe dislocations produced by thermal stresses between glass or alumina fibers and silver chloride, used as a model material for metal matrix composites. Fibers ends are found to punch prismatic loop rows, the length of which is compared with a model presented in Part I of this paper as well as another model based on a mismatching spheroid. The geometry of dislocations punched at fiber ends is discussed for the case where the fiber axis is not coaxial with the glide direction. Elongated loops are also observed to be punched radially by the fiber and their number is correlated to the thermal radial mismatch. A simple model is proposed for the nucleation of such loops, which are usually entangled in a plastic zone surrounding the fiber.
\end{abstract}

\begin{abstract}
Résumé - Une technique de décoration est utilisée pour observer les dislocations produites par tensions thermiques dans un modèle de composite à matrice métallique constitué de fibres de verre ou d'alumine et de chlorure d'argent. Les extrémités de fibre émettent des rangées de boucles prismatiques dont la longueur est comparée à un modèle présenté dans la première partie de cet article ainsi qu'à un autre modèle basé sur un spheroïde incompatible. La géométrie des dislocations émises en bout de fibre est discutée dans le cas où l'axe de la fibre n'est pas coaxial à la direction de glissement. Des boucles allongées émises radialement par la fibre sont aussi observées et leur nombre est corrélé à l'incompatibilité radiale thermique. Un modèle simple est présenté pour la nucléation de ces boucles qui sont habituellement enchevetrées dans une zone plastique entourant la fibre.
\end{abstract}

\begin{abstract}
Zusammenfassung--Mit einem Dekorationsverfahren werden die Versetzungen sichtbar gemacht, die sich wegen thermischer Spannungen zwischen Glas-oder Aluminiumoxidfasern und Silberchlorid bilden; diese Kombination wird als Modell für Verbundmaterialien mit Metallmatrix angesehen. Die Faserenden stoßen Reihen prismatischer Versetzungsschleifen aus; deren Länge wird mit dem in Teil I dieser Untersuchung vorgelegten Modell, wei auch mit einem anderen, auf einem fehlpassenden Ellipsoid beruhenden Modell verglichen. Die Geometrie der an den Fasereneden ausgestoßenen Versetzungsschleifen wird für den Fall diskutiert, daß die Faserachse nicht mit der Gleitrichtung übereinstimmt. Zusätzlich wird beobachtet, daß längliche Versetzungsschleifen von der Faser radial ausgestoßen werden; ihre Zahl hängt mit der thermischen radialen Fehlpassung zusammen. Ein einfaches Modell wird für die Bildung solcher Schleifen, die üblicherweise verschlungen in einer plastischen Zone um die Faser herum verteilt sind, vorgeschlagen.
\end{abstract}

\section{INTRODUCTION}

The plastic relaxation of stresses induced by thermal mismatch of the two constituents of fiber reinforced metals has been studied experimentally using etch pits, slip lines and transmission electron microscopy (TEM). Limitations of these techniques in the investigation of dislocation structures generated in the matrix of metal composites where the reinforcement size is on the order of one, or several, micrometers have been exposed elsewhere [1-3]. It was also shown in these references that several of these limitations can be overcome by the use of silver chloride as a model material for the matrix of fiber reinforced metals. This transparent salt deforms by dislocation mechanisms very similar to those operative in metals. Upon exposure to actinic light, silver chloride photodissociates. The resulting silver preferentially nucleates and grows at crystal defects such as dislocations, grain- and subgrain boundaries, decorating them to a depth of up to $30 \mu \mathrm{m}$ and making them visible to transmission optical microscopy. If the dislocation density is low enough, decorated dislocations can be resolved and observed in the state in which they were during decoration. This technique, developed by Hedges and Mitchell [4], presents great advantages for the imaging of dislocation structures around large inclusions which are difficult to observe by TEM in an undisturbed state. In previous publications, we used silver chloride composites to study thermal mismatch dislocations around glass particles [1] as well as glass microspheres and alumina fibers [2]. We observed tangles of dislocations along the sides of the fibers forming a well-defined plastic zone, as well as rows of prismatic dislocation loops punched at the fiber tip, as observed by Vogelsang et al. [5] in whisker reinforced aluminium using TEM.

In an earlier paper [6] (hereafter referred to as Part I) we analyzed the mechanics of prismatic loop punching at the ends of a thermally mismatching fiber. 
We made use of the shear-lag model proposed by Cox [7] to derive expressions for the fiber longitudinal tensile stress, the interfacial shear stress and the number of prismatic loops punched in the matrix. The length of the resulting row of loops was then determined from the equilibrium of the elastic repulsive stresses between prismatic loops and the lattice friction stress. This model yields different results than a previous one, proposed by Taya and Mori [8], which is based on the assumptions of a misfitting spheroid punching delocalized dislocations. The purpose of this article is to report experimental data on the morphology and length of prismatic loop rows in silver chloride containing glass fibers. We compare the length of loop rows to both models cited above and also present and discuss different dislocation geometries along the sides and tips of fibers.

\section{EXPERIMENTAL}

A detailed description of the experimental procedures has been given in a previous paper [3]. In short, we prepared $0.1 \mathrm{~mm}$ thick plates of silver chloride containing chopped glass fibers (strontia-alumina-silica based), 0.5-4 $\mu \mathrm{m}$ in diameter, purchased from MoSci Corp. (Missouri, U.S.A.), or $3 \mu \mathrm{m}$ diameter alumina fibers, furnished by Imperial Chemical Industries (U.K.). Silver chloride blocks purchased from Engelhard (Ohio, U.S.A.) were purified, filtered and chemically sensitized with 500 ppm cuprous chloride (purchased from Strem Chemicals, Mass., U.S.A.). Flat composite samples were fabricated by squeezing a drop of molten chloride between two quartz plates onto which fibers had been previously placed. The samples were then directionally solidified, and annealed at $673 \mathrm{~K}$ for $2 \mathrm{~h}$. They were finally quenched in flowing argon at a rate of about $1 \mathrm{~K} / \mathrm{s}$ and exposed for $1-2 \mathrm{~h}$ to stroboscopic actinic light. The decorated substructure was recorded photographically using a Zeiss optical microscope in transmission with a $100 \times$ oil immersion lens.

\section{RESULTS AND DISCUSSION}

\subsection{Punching by fiber ends}

3.1.1. Fiber axis parallel to punching direction. In numerous instances, fibers punched a row of loops in the longitudinal directions in a plane parallel to the sample surface (Fig. 1); the row was thus in focus over its entire length. We believe that the fibers which did not exhibit any decorated loops or plastic zone at their tip probably emitted a row of loops at an angle with respect to the sample surface. If the loops were emitted toward the surface, they must have been attracted by it and annihilated; if they were emitted toward a deeper region of the sample, the limited decoration depth attainable with silver chloride $(30 \mu \mathrm{m}$ maximum) would prevent them from being decorated and thus observed in the optical microscope. The relatively large number of fibers which exhibited a row of

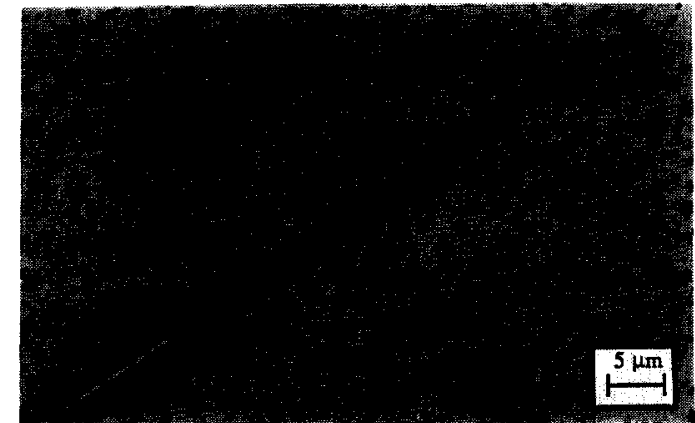

Fig. 1. Row of decorated, prismatic loops emitted by a glass fiber in $\mathrm{AgCl}$. A dark zone of unresolved dislocation tangles surrounds the fiber.

loops at their tips is due, in part, to the fact, reported by Clark and Mitchell [9], that silver chloride crystals grown between quartz plates feature $\{100\}$ planes (and occasionally $\{111\}$ planes) nearly parallel to the quartz plates. Since both these planes are glide planes which contain the glide direction $\langle 110\rangle$, the rows of loops tend to be parallel to the sample surface and in focus over long distances. It was also found that the composite casting procedure resulted in fibers which were parallel to the quartz plates.

The number of individual loops could never be determined with certainty close to the fiber tips, either because the loops could not be resolved or because the decoration was fainter there than at some distance from the fiber, possibly because of a depletion of interstitial silver ions responsible for the decoration, due to the high local dislocation density. In many cases, punched loops extending far away from the fiber encountered a subgrain boundary which blocked them. More loops were then often nucleated on the other side of the boundary to continue the row. Another interesting feature was that a minority of the fibers exhibited a row of loops at both of their ends; when this was the case, the rows were generally of different length.

This last observation suggests that fibers with a single row of loops were either relaxed only on the half length which exhibited a row, or that relaxation of the entire fiber length is possible by emission of a single row. The latter hypothesis requires that the loops of vacancies left at the interface be capable of gliding from one tip to the other in order to relax the fiber half length which did not emit any prismatic loops into the matrix. The two situations are shown schematically in Fig. 2 where only longitudinal strains are considered. The fiber is assumed to be a perfect cylinder, at the surface of which interstitial loops can glide without encountering any obstacle. In practice, the surface of fibers is not perfect and such loops-if they exist in a localized manner-would need to move by a combination of climb and glide to travel along the interface. In a previous publication [2], we reported a similar situation for misfitting glass spheres of diameter $1-3 \mu \mathrm{m}$ in the same silver chloride matrix. 
$\begin{array}{llllllll}6 & 4 & 2 & 0 & 2 & 4 & 6\end{array}$

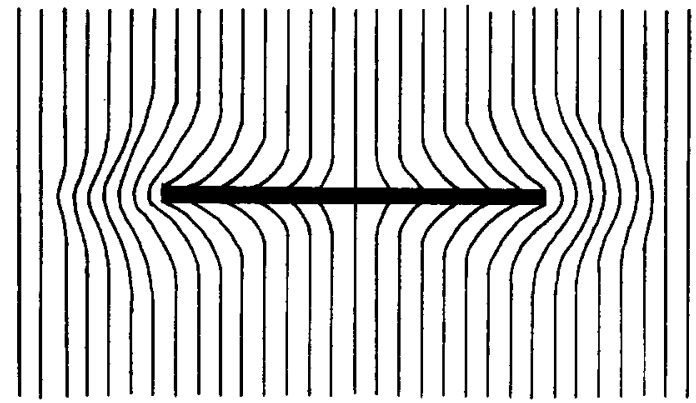

(a)

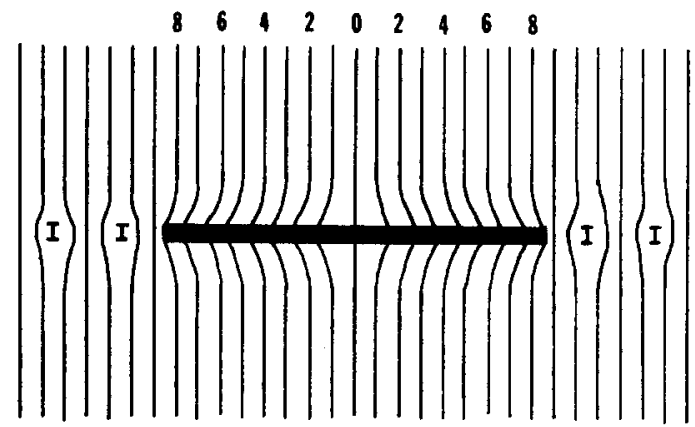

(b)

$\begin{array}{llllllllll}6 & 4 & 2 & 0 & 2 & 4 & 6 & 8 & 10\end{array}$

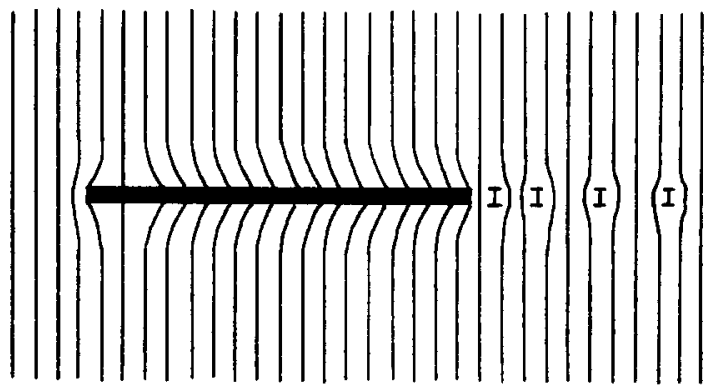

(c)

Fig. 2. Schematics of a mismatching fiber in a crystalline matrix deforming by slip. (a) Fiber under compression and deformed atomic plane in tension before slip (exaggerated deflections for illustrative purpose). (b) Emission of two rows of interstitial loops at both ends and relaxation of the interface by glide of vacancy loops from both ends. The vacancy loops are shown delocalized. Two atomic planes have been added at each ends of the fiber. (c) Emission of a single row of interstitial loops at one end of the fiber and glide of delocalized vacancy loops along the whole interface. The fiber is translated since atomic planes were added only at one end.

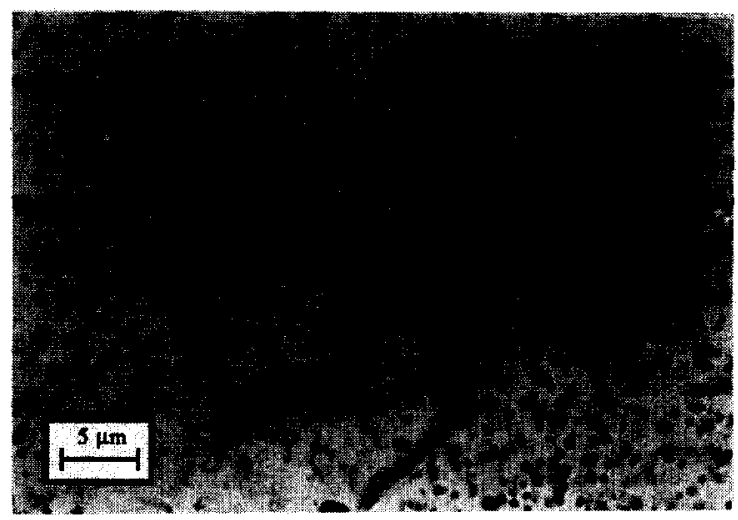

Fig. 3. Glass fiber with punched row of decorated, prismatic loops emitted at an angle in $\mathrm{AgCl}$. The fiber is also surrounded by a plastic zone of unresolved dislocations. 
(a)

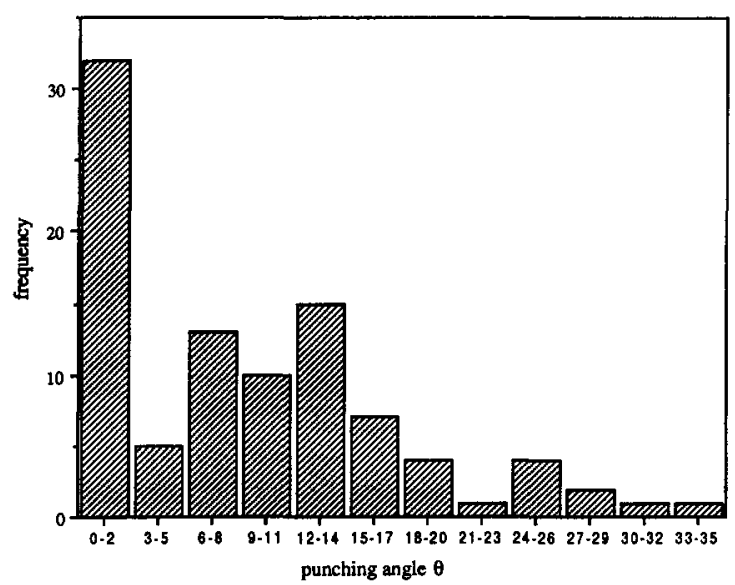

(b)

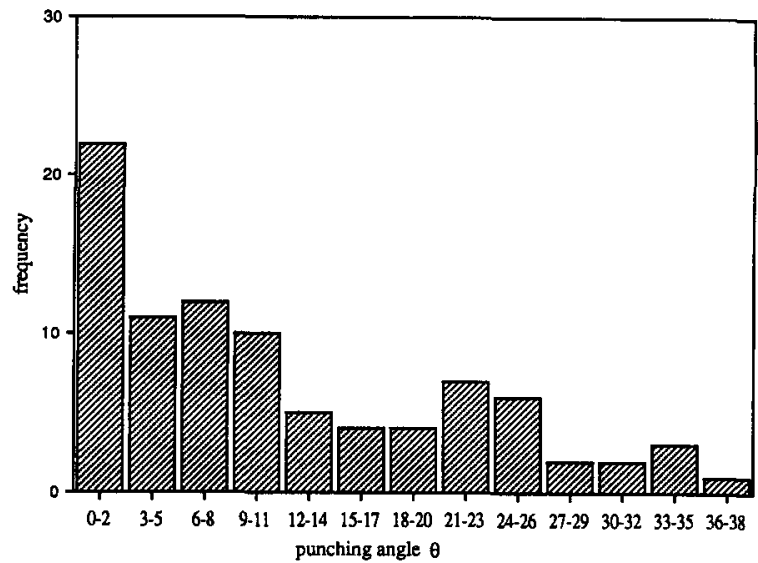

Fig. 4. Plot of the frequency of the angle between the axis of the punched row of loops and that of the emitting fiber in $\mathrm{AgCl}$. (a) Alumina fibers, (b) glass fibers.

These inclusions were often observed to punch loops along a single direction, rather than the 12 necessary for complete relaxation (micrographs published by Mitchell [10] as well as Parasnis and Mitchell [11] show the same phenomenon). In that case too, relaxation can be achieved by motion of the interfacial vacancy loops left at the interface. It is noteworthy that submicroscopic misfitting particles in silver chloride were observed to punch in all crystallographic directions $[3,11]$, leading to the conclusion that the punched dislocation structures depend on the size of the misfitting inclusion.

3.1.2. Fiber axis not parallel to punching direction. Figure 3 shows an example of a fiber which punched a row of loops which is not coaxial with the fiber. Since prismatic loops can only glide along $\langle 110\rangle$ directions, any fiber with an axis making an angle $\theta$ with that direction will only be capable of relaxation by prismatic punching at that angle. The fiber displacement in the longitudinal direction for each loop of

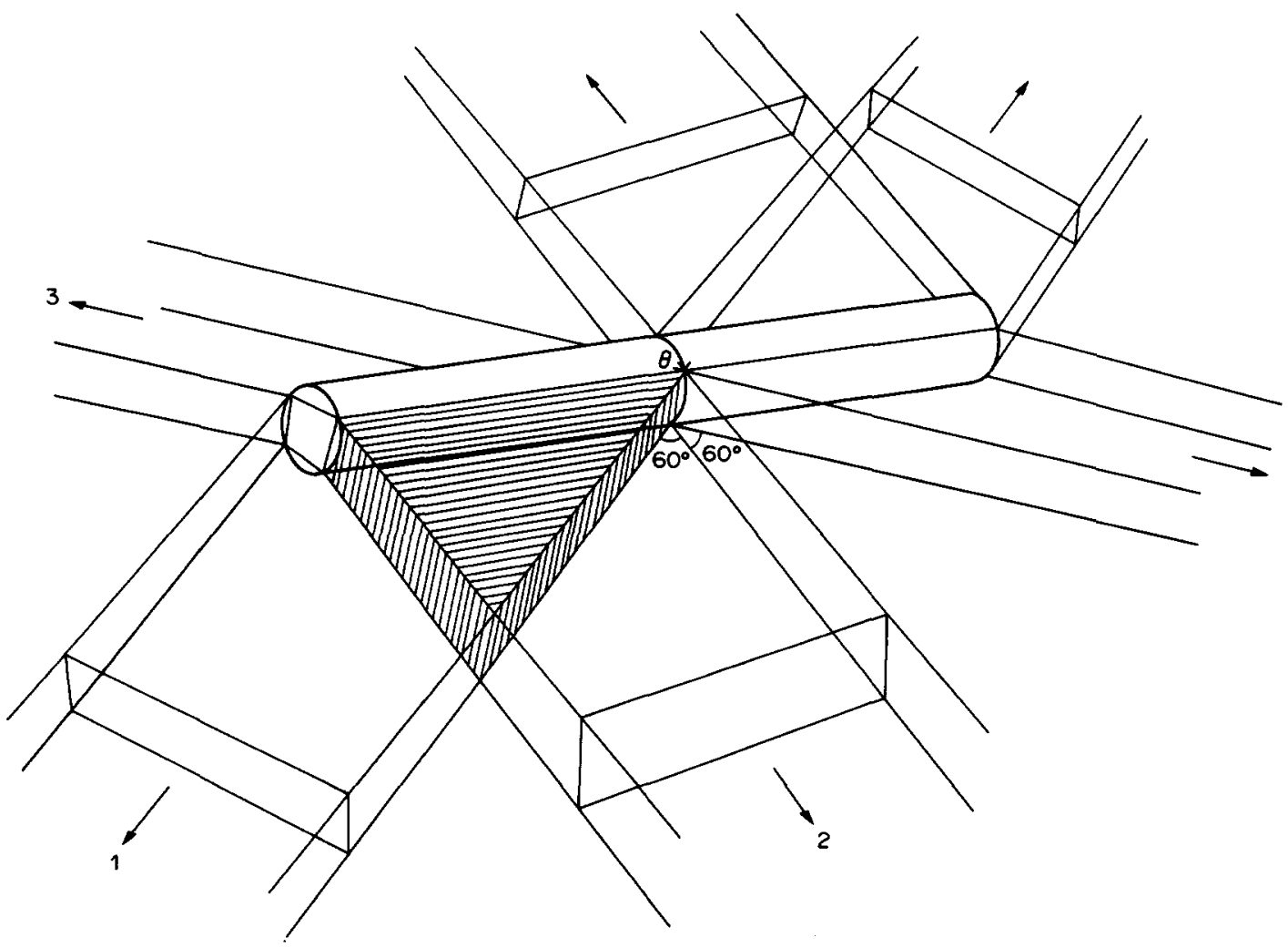

Fig. 5. Schematic of fiber punching elongated dislocation loops along the six $\langle 110\rangle$ glide directions. Cross-hatched volume correspond to the interaction volume between two adjacent glide systems. 
Burgers vector $b$ punched at an angle $\theta$ is equal to the projection of the Burgers vector on the direction of the fiber axis, $b \cdot \cos \theta$. The total number of loops to be punched for a given relaxation is thus increased by a factor $1 / \cos \theta$. Since two adjacent glide directions make an angle of $60^{\circ}$, the maximum punching angle is $30^{\circ}$, if we assume that punching takes place along the direction closest to the fiber axis. Punching at an angle can thus increase the number of loops for a given relaxation by a factor $2 / \sqrt{ } 3$ (i.e. by $15 \%$ ) at most. Such a difference will not significantly alter the punching distance for the relatively large number of loops encountered in these rows [equation (28), Part I].

We counted the frequency of the punching angle $\theta$ and found very few rows punched at an angle larger than $30^{\circ}$ The higher proportion of fibers with small $\theta$ (Fig. 4) is surprising at first, since the fibers are oriented randomly in a plane parallel to the sample surface and the $\langle 110\rangle$ directions are random as well in that plane (but not in space, since the growth of certain $\mathrm{AgCl}$ crystallographic planes is favored, as mentioned above). We can explain this by assuming that entanglement of punched dislocations loop rows reduced the proportion of rows punched at large $\theta$ values.

Consider a portion $L^{\prime}$ of the fiber length, which punches prismatic dislocation loops along all possible directions to relieve both longitudinal and radial strain. This is illustrated in Fig. 5 for the case where $L^{\prime}$ is maximum, equal to the fiber half-length, with the other half-length punching identical loops on the other side of the fiber. This configuration has been observed experimentally, although $L$ ' was generally less than half the fiber length, as in Fig. 6. The intersection between the row relieving longitudinal strain and its neighboring row is the cross hatched volume in Fig. 5. The likelihood of entanglement of punched prismatic dislocations from two adjacent rows punched along $L^{\prime}$ increases as the volume shared by their glide cylinders increases. This intersection volume $V$ is approximately equal to

$$
V=\frac{L^{\prime 2} r}{\sqrt{2}}\left[\frac{1}{\operatorname{tg}(\theta)}-\operatorname{tg}(\pi / 6-\theta)\right]^{-1}
$$

where $r$ is the fiber radius and $\theta$ is the angle between the fiber axis and the closest glide direction. For the range of possible values for $\theta$, from 0 to $\pi / 6$, the intersection volume varies almost linearly between 0 and $L, 2 r / \sqrt{6}$. Entanglement will therefore be more likely at high values of $\theta$. Corresponding rows should therefore be less frequent, as observed in Fig. 4. Observation of elongated loops together with the frequent occurrence of a dark zone of unresolved dislocations along the sides of the fibers (Fig. 6) support this explanation. Figure 7, which gives the frequency of punching a row of dislocation loops at an angle $\theta$ in samples of $\mathrm{AgCl}$ containing elongated glass particles studied in Ref. [1], confirms the findings of Fig. 4 with fibers. Only particles of aspect ratio larger than 2

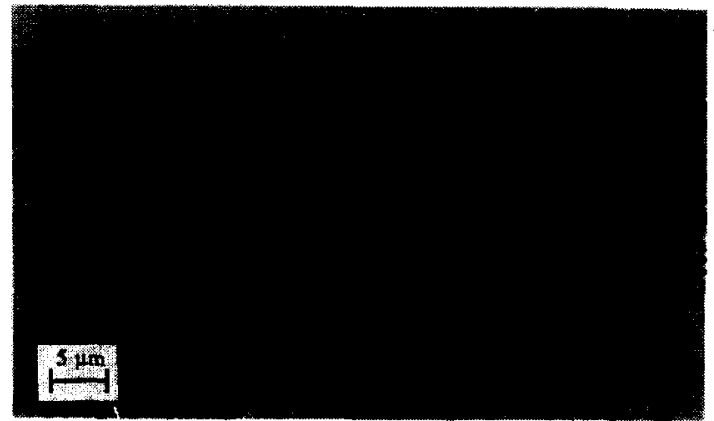

Fig. 6. Glass fiber in $\mathrm{AgCl}$ with decorated dislocation structure consisting of two rows of elongated loops emitted sideways at the fiber ends and a plastic zone surrounding the fiber (dislocations are not resolved). The linear feature is a decorated subgrain boundary.

were taken, the particle axis being that of its largest dimension as seen in the microscope.

In most cases, one punching direction is more favorable than others to relax longitudinal stresses, because it makes the smallest angle with the fiber axis (direction 1 in Fig. 5). However, on occasions, fibers have such orientations with respect to the glide direction that two glide directions form the same angle with the fiber axis and both can be activated simultaneously as in Fig. 8 where the plane of focus is just below the $1 \mu \mathrm{m}$ diameter fiber. The axis of the lower row (A) of elongated loops is parallel to the image plane while that of the upper row (B) forms an angle: the farthest loop from the tip is deepest. Since they are not seen edge-on as the loops in the left row, they appear as ellipses rather than lines. The projected angle between the two rows is about $75^{\circ}$; the actual angle in space is thus most probably $90^{\circ}$, with the two rows punched along non-adjacent $\langle 110\rangle$ glide directions. Such configurations were relatively rare, however.

3.1.3. Punching distance. The length of rows of loops and that of the glass fiber from which they were punched were measured on micrographs. These are

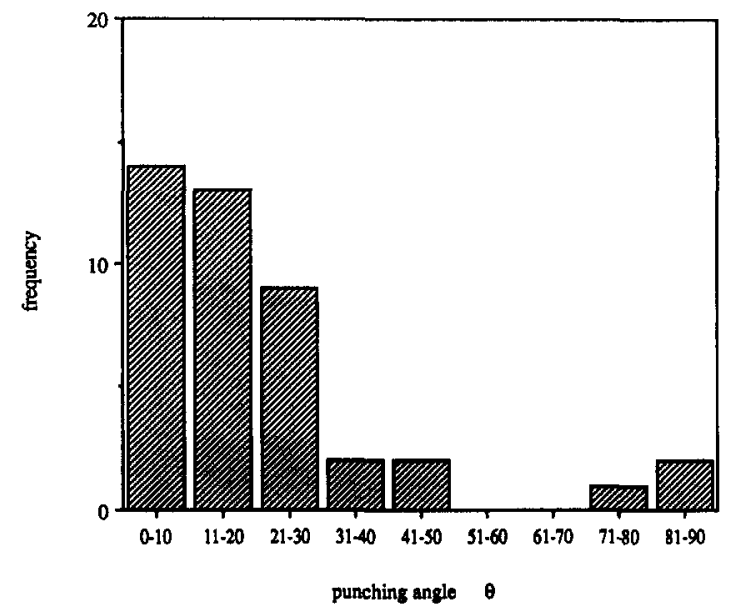

Fig. 7. Plot of the frequency of the punching angle for non-equiaxed glass particles in $\mathrm{AgCl}$. 


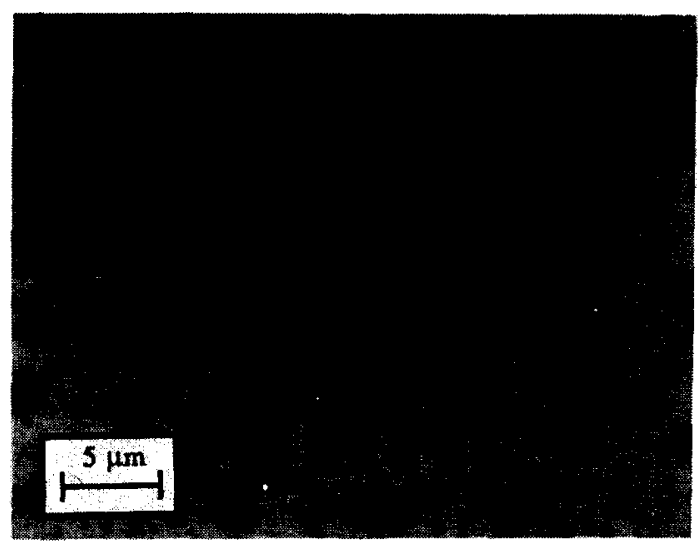

Fig. 8. Two rows of decorated loops punched by the tip of a glass fiber in $\mathrm{AgCl}$. Loops are on non-adjacent glide systems.

plotted in dimensionless form in Fig. 9 for fibers which had punched rows at both their ends, and in Fig. 10 for fibers with a row at only one of their ends. Data are from rows that encountered no visible obstacles (such as other dislocations, subgrain boundaries, fibers). No data are presented for samples with alumina fibers because almost all rows punched had encountered subgrain boundaries (the subgrain size was smaller for these samples than for those with glass fibers). Rows punched at an angle to the glass fibers were included, because the punching angle $\theta$ has only a small influence on the punching distance, as discussed above.

The predictions of the model of Ref. [8] [equations (B1) and (B3) as reported in Part 1] are plotted in Fig. 9 only, since this model is only applicable to fibers punching loops at both tips. Predictions of the model presented in Part 1 [equations (22) and (28) of Part 1] are also plotted in that figure. The thermal and mechanical properties of fibers and matrix used to calculate the theoretical curves are listed in Table 1.
The parameter subject to the largest imprecision is the matrix friction stress, $\tau_{\mathrm{c}}$, the value of which strongly influences predicted punching distances in both models Figure 9 shows curves for $\tau_{\mathrm{c}}=0.5 \mathrm{MPa}$ and $0.25 \mathrm{MPa}$. The former value is the microyield stress in shear measured by Sprackling [12] as well as Haasen and Skrotski [13] and is likely to be an upper bound for the true value of the friction stress for the loops. The lower value of $0.25 \mathrm{MPa}$ is chosen somewhat arbitrarily to illustrate the effect of a smaller, plausible friction stress on the shape and location of the theoretically derived curves. For a value of the friction stress of $0.5 \mathrm{MPa}$, the model of Ref. [8] is somewhat above the range of experimental data [Fig. 9(b)]. For a value of the friction stress of $0.25 \mathrm{MPa}$, predictions from the model of Ref. [8] increase further above the experimental data [Fig. 9(a)]. A generalization of the model of Ref. [8], presented in Ref. [14], shows that assuming concomitant lateral relaxation and allowing for a variable degree of longitudinal relaxation brings theoretical predictions of this model within the error band of the data. Predictions of the model presented in Part I of this work remain within the range of the experimental data for both values of the matrix friction stress.

Equation (22) of Part I was derived for fibers which had punched a row of loops at both their ends [Fig. 2(b)]. For fibers punching at only one end, we assume that nearly the whole interface becomes relaxed as in Fig. 2(c). The number of loops can then be calculated from equation (22) of Part I if we assume that punching at one end of a fiber is equivalent to punching at both ends of a fiber of double length, since in both cases the fiber length being relaxed by a row of loops in the same. Equation (22) of Part I is therefore applied with $L_{\mathrm{f}}$ equal to twice the actual fiber length in Fig. 10. This assumption is not entirely correct since, as shown schematically in Fig. 2(c), a backstress is exerted by the matrix on the fiber end having not punched, in order to equilibrate the net
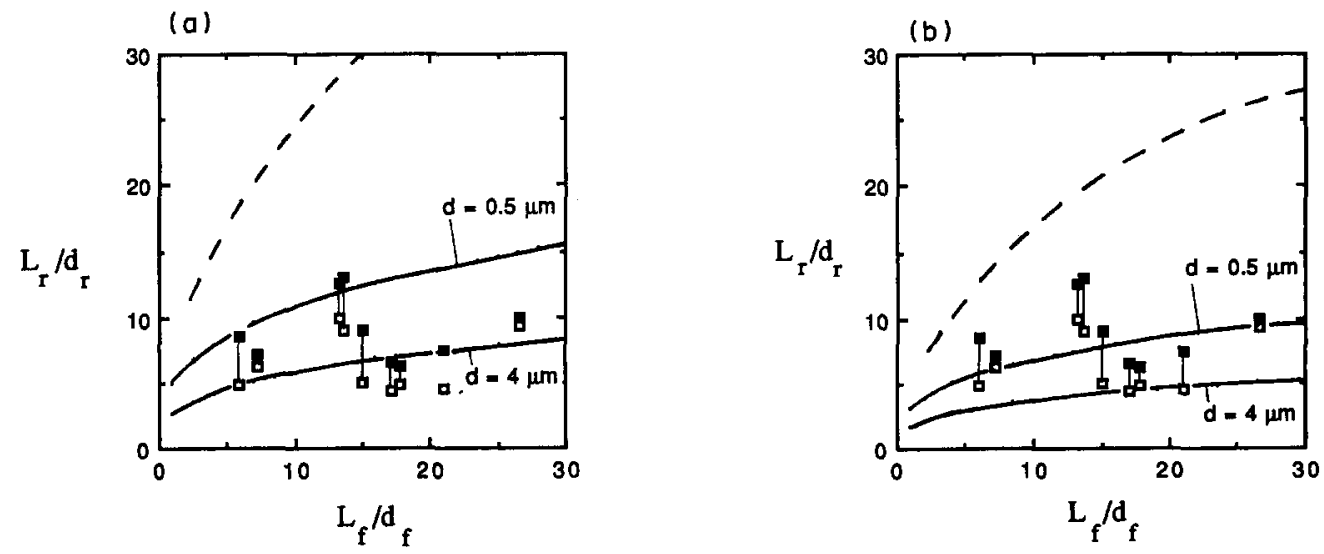

Fig. 9. (a) Plot of the row length (divided by its diameter) vs the fiber aspect ratio. Squares represent experimental data for glass fibers of diameter between 0.5 and $4 \mu \mathrm{m}$ which punched rows at both their ends; the longer and shorter rows for a given fiber are represented by connected filled and empty symbols respectively. The full lines are calculated from equations (22) and (28) (Part I) and dotted lines are calculated from equations (B1) and (B3), reported in Part I, according to Ref. [8], for a value of the friction stress of $0.25 \mathrm{MPa}$. (b) Same as (a), but for a value of the friction stress equal to $0.5 \mathrm{MPa}$. 
force produced by the asymmetrical distribution of interfacial shear stress. For this assumption to be correct, the length of fiber over which this reactive elastic force is exerted must be small compared to the total fiber length.

Predicted punching distances from equations (22) and (28) of Part I fall within the scatter in experimental data points, and predict trends in agreement with those of the band of experimental data points. Scatter in experimental data can have several origins. As mentioned above, the lower data points can be explained by the blocking of rows of loops by obstacles not visible in the microscope, such as unresolved dislocations or submicroscopic inclusions. This effect should become stronger the longer the row, because of the higher probability of encountering an obstacle. Extraneous long-range stresses might also induce scatter in the data points, both elongating and shortening

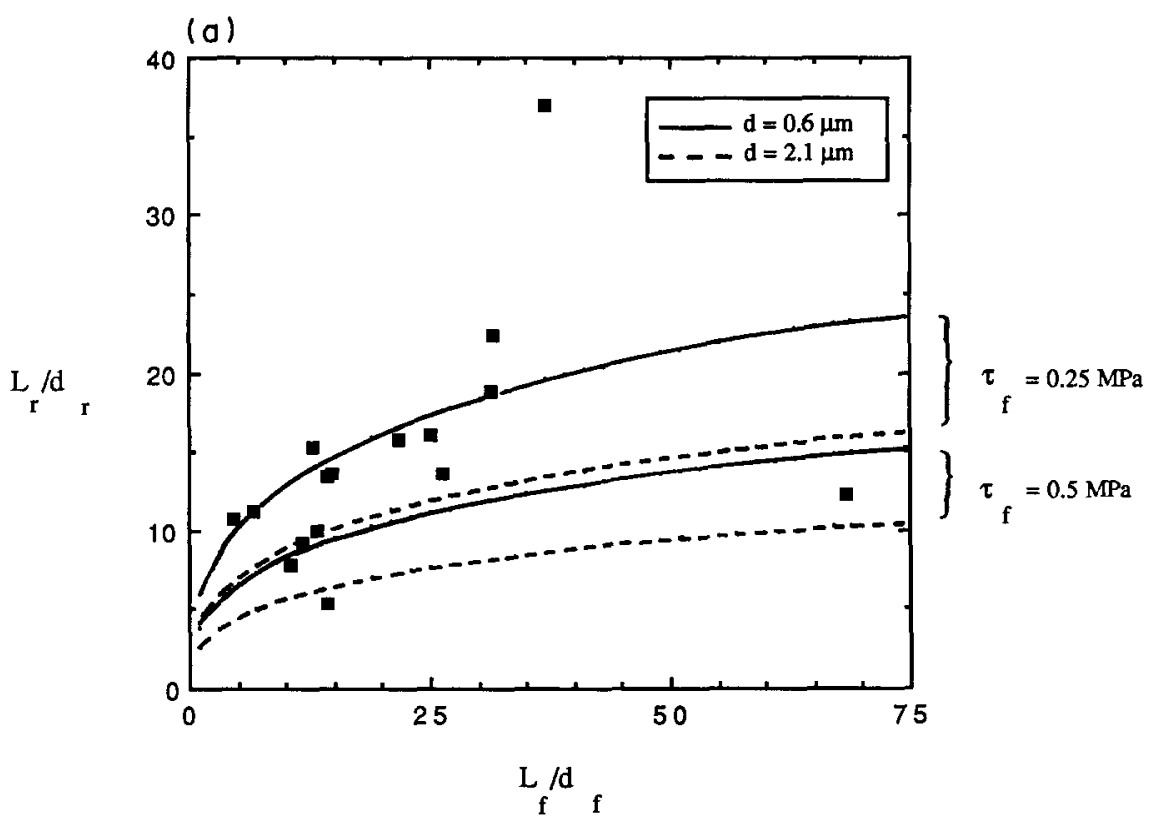

(b)

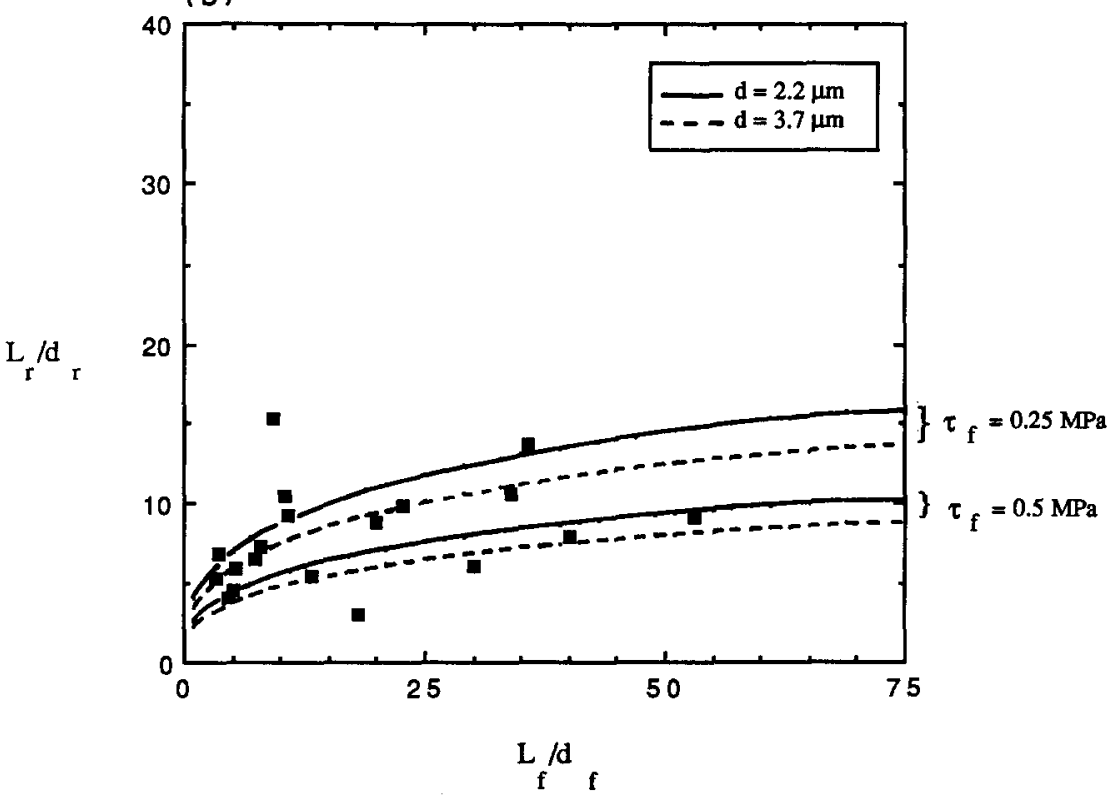

Fig. 10. (a) Plot of the row length (divided by its diameter) vs the fiber aspect ratio. Squares represent experimental data for glass fibers with a diameter between $0.6 \mu \mathrm{m}$ and $2.1 \mu \mathrm{m}$ which had punched a row at only one of their tips. The lines are calculated from equations (22) and (28) of Part I for the two above diameters and a friction stress of 0.25 and $0.5 \mathrm{MPa}$. (b) Same as (a), but for glass fibers with a diameter between 2.2 and $3.7 \mu \mathrm{m}$. 
Table 1. Thermal and mechanical properties of matrix and fibers

\begin{tabular}{lccc}
\hline & $\mathrm{AgCl}$ & $\mathrm{Al}_{2} \mathrm{O}_{3}$ & Glass \\
\hline Elastic modulus $(\mathrm{GPa})$ & & $300^{\mathrm{a}}$ & $85^{\mathrm{a}}$ \\
Shear modulus $(\mathrm{GPa})$ & $5.5^{\mathrm{b}}$ & & \\
Poisson's ratio & $0.343^{\mathrm{c}}$ & $0.24^{\mathrm{d}}$ & $0.22^{\mathrm{d}}$ \\
CTE $\left(10^{6} \mathrm{~K}^{-1}\right)$ & $30^{\mathrm{c}}$ & $9^{\mathrm{a}}$ & $6.5^{\mathrm{a}}$ \\
Volume fraction & & 0.01 & 0.01 \\
Burgers vector $(\mathrm{nm})$ & $0.384^{\mathrm{c}}$ & & \\
Friction stress $(\mathrm{MPa})$ & $0.5^{\mathrm{c}}$ & & \\
$\Delta T[\mathrm{~K}]$ & $100^{\mathrm{f}}$ & & \\
\hline
\end{tabular}

a: manufacturer information; b: calculated for [110] directions from elastic constants reported in Ref. [21]; c: Ref. [21]; d: Ref. [22]; e: Ref. [12] and [13]; f: Ref. [2].

the measured row length. Finally, an effect which might systematically increase the row length is the electrostatic repulsion between charged loops. As discussed in greater detail in the Appendix, this is not expected to induce forces larger than the elastic repulsion on which the calculations are based and thus can probably be neglected. If, however, the first few loops of the train are charged to the maximum possible extent, this effect might produce an increase of row length by typically $1-10 \mu \mathrm{m}$. Given the experimental errors in the measurements cited above as well as the uncertainties connected with some of the physical constants used in the model (the lattice friction stress, the critical interfacial shear stress assumed to be equal to the lattice friction stress, and the temperature excursion $\Delta T$ of $100 \mathrm{~K}$ which has an experimental error of typically $30 \mathrm{~K}$ [2]) and the simplifying assumptions made in the model presented in Part I (perfect dislocation nucleation, cylindrical inclusion with smooth surface, no radial mismatch, etc.), we feel that the agreement between the data and our model is satisfactory.

The aspect ratio of the fibers investigated here are significantly below the critical aspect ratio above which the model of Ref. [8] predicts suppression of punching, in disagreement with the predictions of the model in Part I. While this latter model predicts a finite, non-zero, maximum value of the punching distance for fibers of infinite aspect ratios, curves calculated with the model of Ref. [8] in Fig. 9 reach a maximum and drop to zero for a critical aspect ratio which has a value of 460 for $\tau_{c}=0.5 \mathrm{MPa}$ [Fig. 9(b)] and 930 for $\tau_{\mathrm{c}}=0.25 \mathrm{MPa}$ [Fig. 9(a)]. Experimental difficulties prevented us from obtaining significant data with such long fibers: fibers are supplied in a matte from which they are difficult to extract without breaking them and long fibers require a large region of matrix free of sources of interaction such as subgrain boundaries. The largest aspect ratio found in our samples was for a glass fiber, $580 \mu \mathrm{m}$ long, which had an aspect ratio of 644 , and punched a single row of loops of length $28 \mu \mathrm{m}$. This row was not included in data plotted in Fig. 10 because its loops had varying diameters (as for the outward row of the long fiber of Fig. 11). Using the fiber diameter of $0.9 \mu \mathrm{m}$ in equation (22) of Part I and the average loop diameter of $2.4 \mu \mathrm{m}$ in equation (28) of Part 1, we predict a loop row length of $20 \mu \mathrm{m}$ for $\tau_{\mathrm{c}}=0.5 \mathrm{MPa}$ and $31 \mu \mathrm{m}$ for $\tau_{\mathrm{c}}=0.25 \mathrm{MPa}$. The model of Ref. [8] predicts for a spheroid of aspect ratio 644 row lengths of 0 and $44 \mu \mathrm{m}$, respectively. We emphasize that this data point is the only one we collected, and is therefore not conclusive.

3.1.4. Punching of pairs of rows. In numerous cases, we observed a pair of coaxial rows, located on either side of the tip of a fiber as illustrated by Fig. 11 . These rows were only found at the ends of fibers and the far side of the elongated dislocations was always emanating from the fiber tip. The outward row was completely separated from the fiber and longer than the inward row, the dislocations of which were always connected to the fiber. While it is quite safe to assume that the outward row is not different from the ones described in Section 3.1.2, the nature of the inward row is less clear. It is clearly different from the plastic zone of tangled dislocations usually found along the sides of fibers (Fig. 6). Assuming that they are prismatic, the dislocations in the inward row are unlikely to be interstitial since their number would be too high to be generated by radial misfit [see equation (2) in the following section] and since the orientation of their Burgers vector would be less favorable than other glide directions with a Burgers vector closer to the radial direction. Also, if the row were produced by radial misfit, it would occur at other places along the fiber rather than solely at its tip. We propose the hypothesis, explained in more detail in what follows, that the inward row is constituted of vacancy loops formed at the same time as the interstitial loops of the outward row.

Every time a prismatic interstitial loop is created at the interface of a misfitting particle, a vacancy loop of same size is formed by conservation of matter. In most cases, this vacancy loop is not further considered because it becomes delocalized while it is absorbed at the interface, i.e. the volume of the vacancy loop is replaced by the same volume of the expanding inclusion [Fig. 2(b)]. When loops are punched at an angle from the fiber axis, the punched prismatic interstitial loop can be larger than the projection, along its glide direction, of the fiber crosssection. For this reason, the volume of each vacancy loop accompanying interstitial loop emission cannot be entirely taken up by the expanding fiber. A smaller vacancy loop then remains after punching of a prismatic interstitial loop, of volume equal to the difference of the volume of the interstitial loop and that taken up by expansion of the inclusion. This process is schematically drawn in Fig. 12. When further pairs are formed on each side of the inclusion, loops within a row will repel each other while loops from different rows will attract each other. The resulting perturbation of the length of the row of interstitial loops was calculated in Ref. [15], and was found to be small.

While the limited resolution available in silver chloride allows to draw few conclusions concerning the nucleation of such interstitial and vacancy loops, 


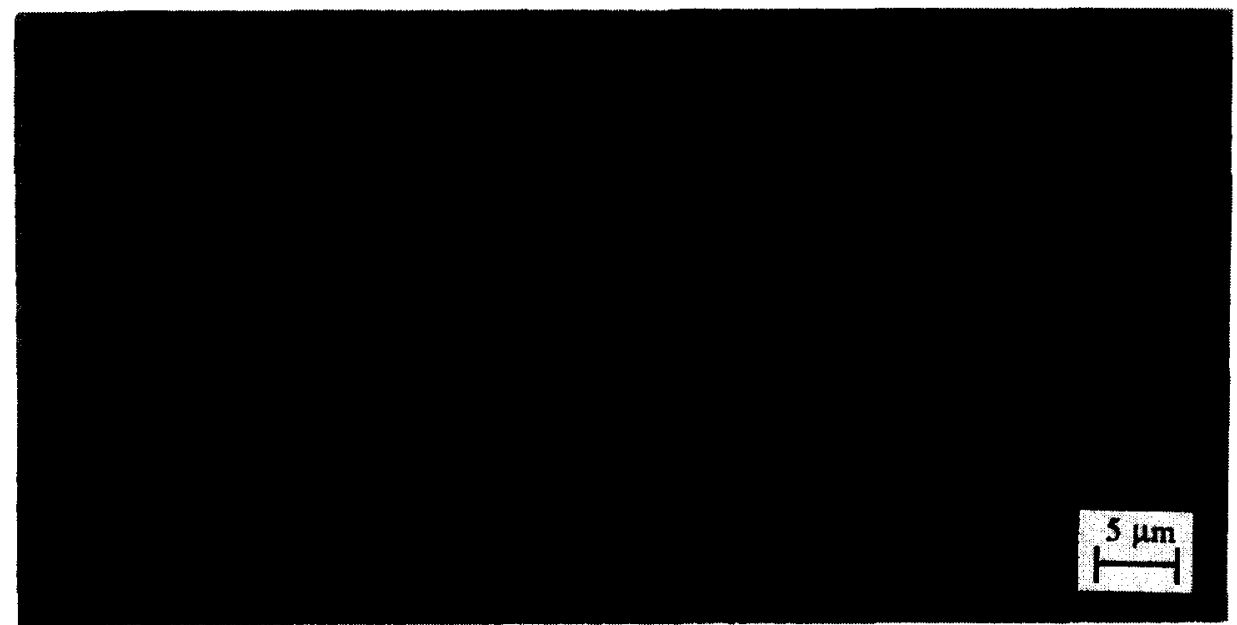

Fig. 11. Two glass fibers in $\mathrm{AgCl}$ showing the same dislocation structure: a double row of decorated, elongated loops emitted by the tip of the fiber. The outward row is similar in nature to that in Fig. 3; the inward row is attached to the fiber.

it is noteworthy that the configuration proposed above would indeed relax the longitudinal-and to some extent the radial-mismatch between the fiber and the matrix. Figure 12 illustrates that point, showing the atomic planes before and after creation of the loop pair. While atomic spacings are widely exaggerated for clarity, it is clear that the vacancy loop induces some relaxation at the tip of the fiber, as does the presence of the interstitial loop on the other side of the fiber tip. It is also apparent by observing

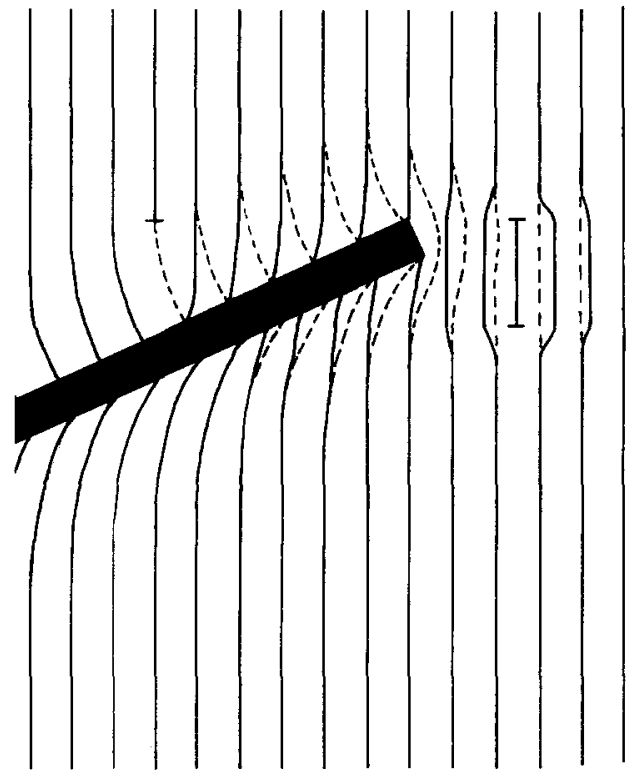

Fig. 12. Schematic drawing proposed as an explanation for the structure of Fig. 11. Dotted lines: atomic plane stressed by the mismatching fiber before relaxation. Full lines; atomic planes after creation of a pair of prismatic interstitial and vacancy loops on either side of the fiber. The interstitial loop glides away from the fiber while the vacancy loop stays attached to the fiber for maximum relaxation of the interface. Atomic plane deflections are exaggerated for clarity. shear strains in Fig. 12 that one end of the vacancy loops will tend to stay in the region of maximum atomic plane disregistry, i.e. close to the fiber tip, as observed experimentally.

\subsection{Punching by fiber sides}

Figure 13 shows a fiber $A$ which punched loops at both its tips at a low angle. The shorter fiber B also punched loops at its tips, as well as longer dislocations along its sides which glided in the same direction as that of the row punched by fiber $\mathbf{A}$. This observation was made in many occasions and lends credence to the hypothesis that the side dislocations of fibre B are elongated prismatic loops punched according to the simple model shown in Fig. 5, where the fiber is oriented along a $\langle 111\rangle$ direction. The sides of fiber A are surrounded by a dark plastic zone formed of dislocations which probably became entangled before they could glide away from the interface, as in the case of fiber B. Fiber $\mathrm{C}$ also has elongated dislocations on its sides (some of which can be resolved) forming a dense plastic zone. It can be deduced from the angle of $90^{\circ}$ between the glide directions in this particular subgrain that the image plane is parallel to a $\{100\}$ plane. Figure 14 shows a similar situation in which elongated loops seem to have emanated from the tip of the fiber, as is to be expected due to the stress concentration at the sharp corner of the chopped fibers.

To explain these observations, we propose to extend to a cylindrical inclusion the model presented by Ashby and Johnson [16] whereby a shear loop nucleates at the surface of a sphere and produces a prismatic loop by cross-slip of its screw components. Figure 15(a) shows such a shear loop nucleated at the edge of the fiber tip, which is a region of stress concentration. The fiber axis is assumed to be parallel to a $\langle 111\rangle$ direction. While the edge component of the shear loop glides radially away from the fiber due 


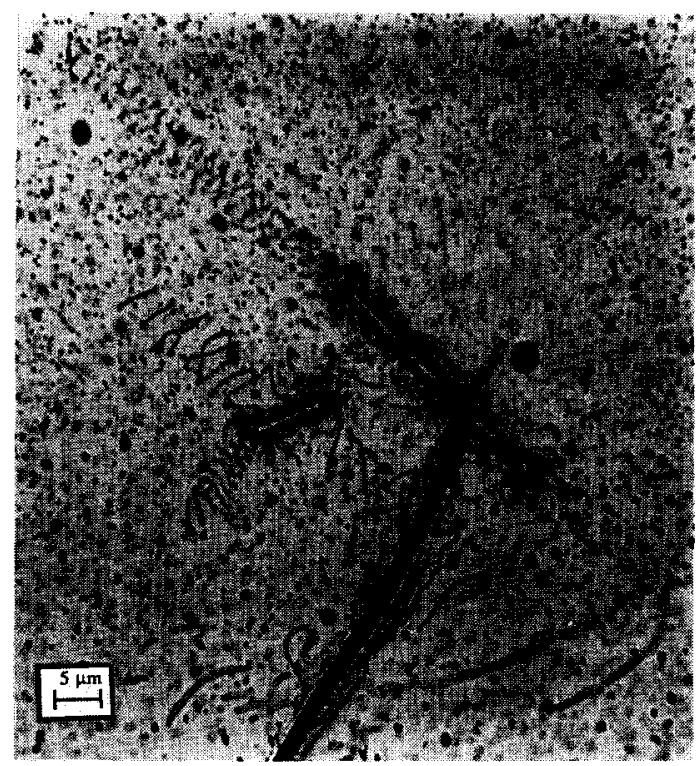

Fig. 13. Three glass fibers having emitted decorated dislocations in $\mathrm{AgCl}$. Fiber A punched a row of loops at each of its end. Fiber $B$ punched four rows, two of which are blocked by fiber $A$ and $C$. Fiber $C$ is surrounded by a plastic zone in which some dislocations can be resolved.

to the stress field, the screw components of the loop cross-slip along the sides of the fiber, "unzipping" in the process a long prismatic loop. The movement stops when the end of the fiber is reached or when the screw components are blocked by obstacles. Another possibility is represented in Fig. 15(b) where a long shear loop is nucleated (for example because of fiber surface roughness) so that its edge portion is parallel to the fiber axis. Again, screw components glide and annihilate to form a prismatic loop. Both Figs 14 and 16 (as well as many others not included in this article) show dislocation structures which can be explained by such punching. In particular, these figures show dislocations with one end still attached to the fiber, while the other has glided away from it. Elongated dislocations were, however, only observed along fibers

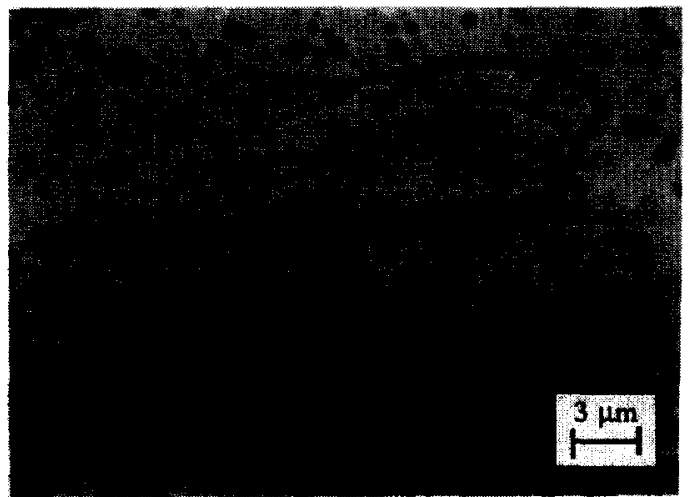

Fig. 14. Glass fiber in $\mathrm{AgCl}$ with elongated, decorated loops emitted by its sides. A dislocation is still attached and bowed due to the residual fiber stress. with the smallest diameters: for fiber diameters larger than about $1.2 \mu \mathrm{m}$, the dislocations were without exception entangled and formed a dense plastic zone such as that visible in Fig. 17. This is presumably because more dislocations are necessary to relax the radial stresses and the glide system intersection volumes increase with increasing fiber diameter, both leading to entanglement. We have not extended to cylinders the energy calculations of Ashby and Johnson [16], who predicted the range of misfit strains and radii for which spheres nucleate prismatic dislocations, because stress concentration at the tip edge and the orientation relationship between the fiber and the slip directions are significant complicating factors.

Considering only radial stresses, the number of elongated prismatic dislocations punched by the sides of the fiber can be predicted using the simple geometric model of Fig. 5. The prismatic dislocations are assumed to nucleate on the fiber surface where the resolved shear stress is maximal, resulting in a dislocation height of $d / \sqrt{ } 2$, where $d$ is the fiber diameter. The thickness of the region of fiber that the dislocation relaxes thus varies between $d / \sqrt{2}$ at the nucleation point and $d$. Assuming that the mismatch strain $\Delta \alpha \Delta T$ is totally relaxed by the emission of $n$ loops of Burgers vector $b$ forming an angle $\theta$ with the fiber radial direction, we can write

$$
n=\frac{\Delta \alpha \Delta T d}{\sqrt{2} b \cos \theta}
$$

where $\Delta \alpha$ is the coefficient of thermal expansion difference between the matrix and the fiber and $\Delta T$ is the temperature excursion where slip is operative. The maximum number of loops $n_{\max }$ is reached for the maximum angle $\theta=30^{\circ}$ (as discussed above) and by replacing the factor $\sqrt{2}$ by 1 in equation (2), i.e. considering the maximum mismatch of the fiber at its largest dimension $d$. Equation (2) is plotted in Fig. 18, using the materials values listed in Table 1 for the fiber and the matrix as well as the previously determined value of $\Delta T=100 \pm 30 \mathrm{~K}$ [2]. In this figure, we have also plotted experimental values for the maximum number of dislocations emitted radially at any point along the length of each fiber. The experimental points fall on or below the upper bound calculated above, as expected if the simple geometric model presented above (Fig. 5) is correct. In gathering the experimental data, we counted the dislocation lines on both sides of the fiber. Usually, one side had punched significantly more dislocations than the other. The average number found along each fiber was usually smaller than this maximum by $25-50 \%$. We only considered fibers with dislocations far away from the tip to minimize the effect of longitudinal stresses and stress concentrations.

Around most fibers, dislocations were tangled in a plastic zone around the fiber (Fig. 17) and could not be reliably counted. Figure 16 shows an example where the side dislocations can be resolved and 
(a)

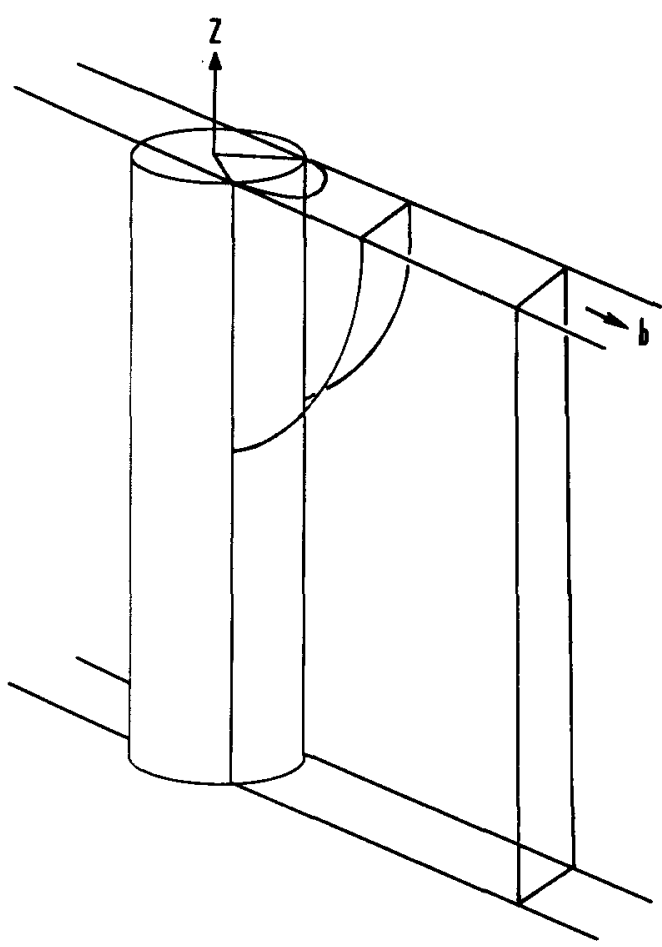

(b)

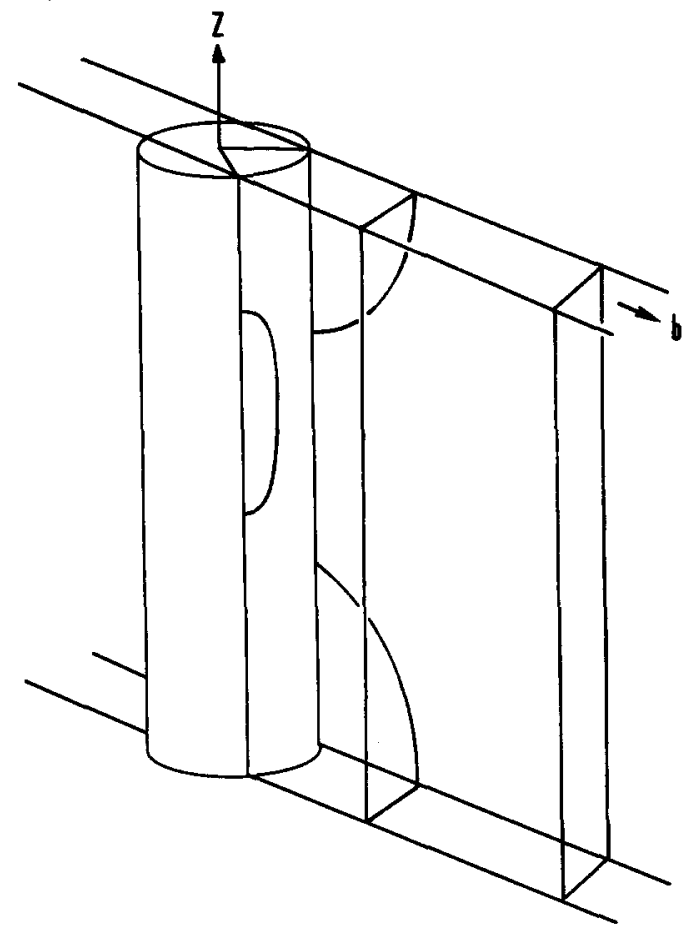

Fig. 15. (a) Proposed mechanism of nucleation of prismatic loops to explain structure in Figs 14 and 16. Three stages are shown: (i) formation of a shear loop at fiber end or irregularity, (ii) cross-slip of screw segments and (iii) fully formed prismatic loop. (b) Variation of (a), with formation of the shear loop on another glide plane.

counted because they glided a considerable distance from the fiber. A small dark zone is still visible around the fiber and might be due to dislocations relaxed along other directions. Another interesting feature, visible in Figs 14, 16 and 17 and reported earlier for alumina fibers [2], is the pinning of dislocations which are bowed away from the glass fiber, probably because

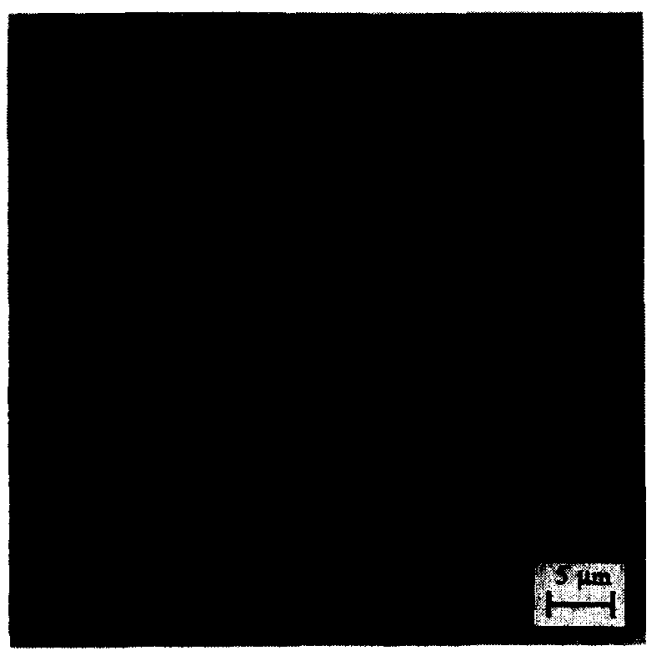

Fig. 16. Long, decorated loops emitted by the sides of a long glass fiber in $\mathrm{AgCl}$. Some loops are still attached to the fiber and all are bowed by its residual stress. of residual elastic stresses near the fiber. The radius of curvature of the dislocations, which is inversely proportional to the stress on the dislocation, increases with increasing distance from the fiber, in accord with the above interpretation.

\section{SUMMARY}

Silver chloride containing small glass or alumina fibers is used as a model material to study plasticity in fiber reinforced metals. The dislocations resulting from the mismatch of thermal expansion between the inclusions and the matrix are made visible to transmission optical microscopy by bulk decoration through photodissociation of the matrix at ambient temperature.

Rows of prismatic loops are punched by the fiber tip to relax the large longitudinal thermal mismatch of the fiber. The length of the row is found to increase with that of the fiber. Reasonable agreement is found between the experimental data and a theoretical model proposed in Part 1 of this work.

When the fiber axis is not parallel to a glide direction, elongated prismatic dislocations are punched by the sides and the tips of the fiber. The probability of forming long rows of loops is higher when the fiber axis forms a small angle with the glide direction. This is because the volume in which loops of adjacent glide directions can interact and become entangled 


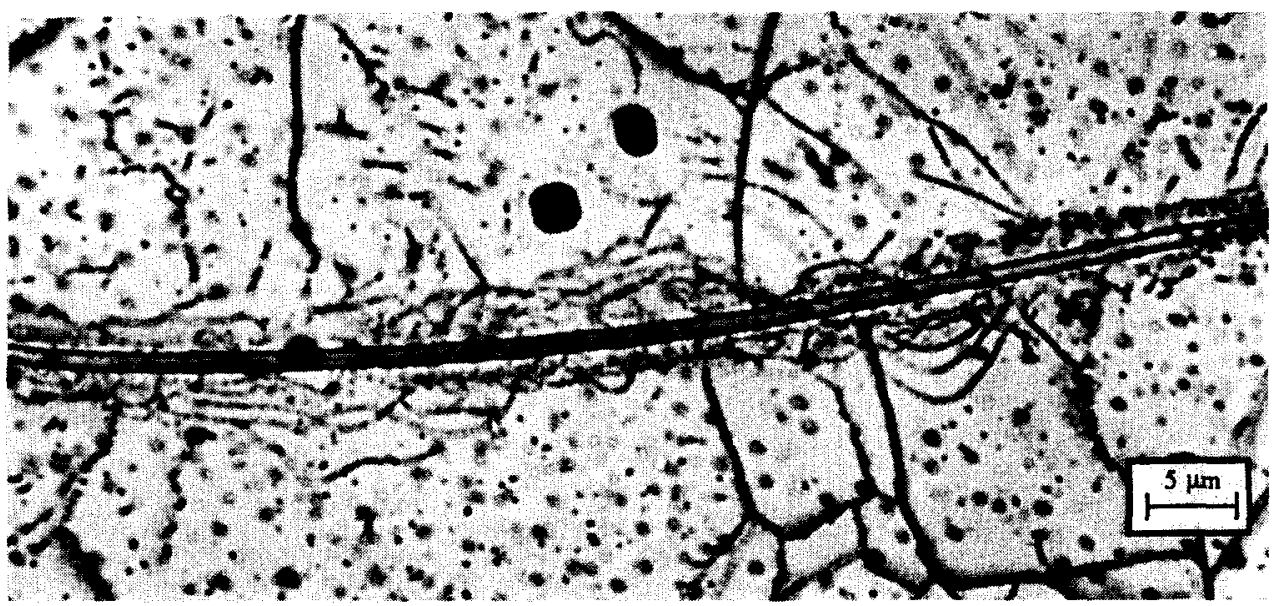

Fig. 17. Decorated dislocations forming the tangled plastic zone surrounding a long glass fiber in $\mathrm{AgCl}$. Some of the dislocations can be resolved.

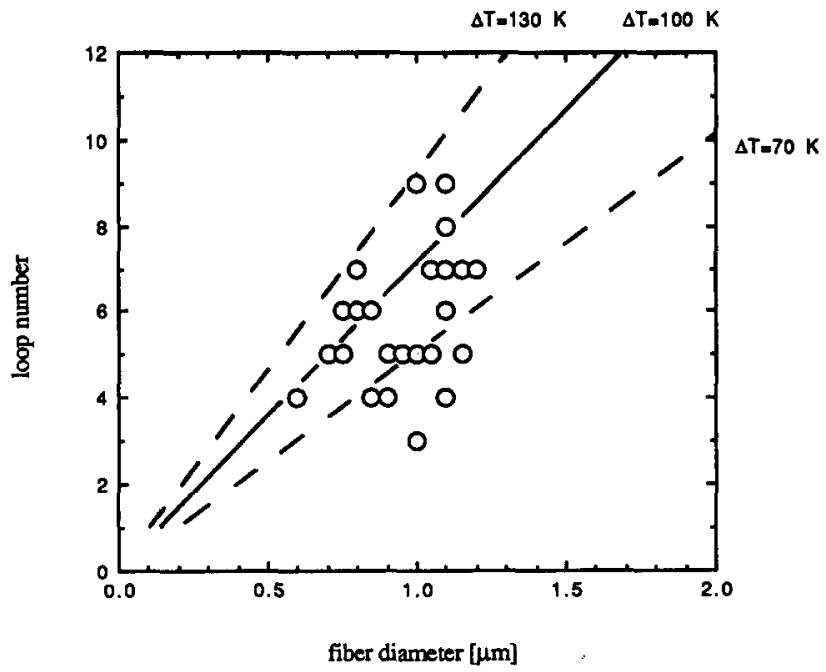

Fig. 18. Plot of the maximum number of loops emitted radially by the sides of glass fibers as a function of the fiber diameter. Circles represent experimental data and lines are calculated from equation (2) for different values of $\Delta T$.

decreases with decreasing angle. Certain fibers are found to punch a pair of coaxial rows of prismatic loops on either sides of their tip. We propose that these rows are formed of interstitial and vacancy loops respectively.

Elongated dislocation loops can be punched radially by the sides of the fiber and usually form a tangled plastic zone. When the fiber diameter is small, these loops form a pile-up and can be counted; their number is correlated with the radial mismatch estimated from known geometrical and thermal parameters of the fiber and the matrix. Based on microstructural observations, we explain nucleation of punched loops by extending to cylinders the loop punching model proposed by Ashby and Johnson [16] for spheres.

Acknowledgement-This work was supported by the National Science Foundation, grant No. DMR-9002558.

\section{REFERENCES}

1. D. C. Dunand and A. Mortensen, Mater. Sci. Engng A. In press.

2. D. C. Dunand and A. Mortensen, Acta metall. mater. 39, 127 (1991).

3. D. C. Dunand and A. Mortensen, Mater. Sci. Engng A. In press.

4. J. M. Hedges and J. W. Mitchell, Phil. Mag. 7, 44, 223 (1953).

5. M. Vogelsang, R. J. Arsenault and R. M. Fisher, Metall. Trans. 17A, 379 (1986).

6. D. C. Dunand and A. Mortensen, Acta metall. Mater. 39 (1991). In press.

7. H. L. Cox, Br. J. appl. Phys. 3, 72 (1952).

8. M. Taya and T. Mori, Acta metall. 35, 155 (1987).

9. P. V. Clark and J. W. Mitchell, J. Phot. Sci. 4, 1 (1956).

10. J. W. Mitchell, Growth and Perfection of Crystals, p. 386. Wiley, New York (1958).

11. A. S. Parasnis and J. W. Mitchell, Phil. Mag. 8, 4, 171 (1959).

12. M. T. Sprackling, Phil Mag. 13, 1293 (1966). 
13. P. Haasen and W. Skrotski, J. Physique 42, C3-119 (1981).

14. D. C. Dunand and A. Mortensen, Scripta metall mater. 25, 761 (1991).

15. D. C. Dunand and A. Mortensen, Scripta metall. mater. 25, 607 (1991).

16. M. F. Ashby and L. Johnson, Phil. Mag. 20, 1009 (1969).

17. M. T. Sprackling, The Plastic Deformation of Simple Ionic Crystals. Academic Press, New York (1976).

18. P. L. Pratt, Vacancies and Other Defects in Metals, Inst. Met. at AERE, Harwell, p. 99 (1958).

19. L. M. Brown, Physica status solidi 1, 585 (1961).

20. C. Kittel, Introduction to Solid State Physics, 4th edn. Wiley, New York (1971).

21. Gmelins Institut Editor, Gmelins Handbuch der anorganischen Chemie: Silber B1, Verlag Chemie (1971).

22. A. Kelly and N. H. Macmillan, Strong Solids. Oxford Science Publ. (1986).

\section{APPENDIX \\ Estimation of the Electrostatic Interaction Between Loops}

Edge dislocations in ionics carry a net charge due to an excess of charged jogs of one sign formed by the absorption by the dislocation line of point defects [17]. At equilibrium, the electroneutrality is preserved because a sheath of defects of the other sign surrounds the dislocation [18]. A fast moving dislocation may however display a net long range charge if it breaks away from the cloud of compensating point defects [19]. The line charge density of edge dislocations in $\mathrm{NaCl}$ has been measured by many investigators as $10^{-12}-410^{-11} \mathrm{C} / \mathrm{m}$, but no such information could be found for $\mathrm{AgCl}$. A rough estimation of the line charge can be made by assuming that it is proportional to the cubic root of the equilibrium defect concentration. The ratio of the defect concentration at $300 \mathrm{~K}$ in $\mathrm{AgCl}$ to that in $\mathrm{NaCl}$ is 4250 [20], the cubic root of which is 16 , leading to a dislocation line density for $\mathrm{AgCl}$ in the range of $2 \cdot 10^{-11}-8 \cdot 10^{-10} \mathrm{C} / \mathrm{m}$. Another significant difference between $\mathrm{NaCl}$ and $\mathrm{AgCl}$ is the nature of the predominant point defects: Schottky-type for $\mathrm{NaCl}$ and Frenkel-type for $\mathrm{AgCl}$. Defect mobility is also much higher in $\mathrm{AgCl}$.

Assume that the charges on a loop are spread equally along its circumference. This is an acceptable assumption since the typical distance between two loops is significantly larger than the distance $\Delta$ between two jogs of charge $e / 2$ on a loop, given by

$$
\Delta=\mathrm{e} / 2 \rho_{q}
$$

where $\rho_{q}$ is the line charge density. Using the line density values estimated above equation (A1) yields a value for $\Delta$ in the range of $0.1-4 \mathrm{~nm}$. The lower value, corresponding to a line density of $8 \cdot 10^{-10} \mathrm{C} / \mathrm{m}$, is clearly unreasonable since it is less than the interatomic distance in $\mathrm{AgCl}$. We will thus assume an upper value of $10^{-10} \mathrm{C} / \mathrm{m}$ for the line density. In what follows, we make a rough approximation of the repulsive electrostatic force between punched prismatic loops. This force must be added to the elastic repulsive force existing between the loops.
The force $F_{q}$ between the two charged loops separated by a distance $L$ is found by Coulomb's law

$$
F_{9}=k q^{2} / L^{2}
$$

where $k=9 \cdot 10^{9} \mathrm{Nm}^{2} / \mathrm{C}^{2}$ and $q$ is the total charge carried by one loop of radius $r$ :

$$
q=2 \pi r \rho_{q} .
$$

The force $F_{q}$ is equivalent to an elastic shear stress $\tau_{q}$ on the loop

$$
\tau_{q}=F_{q} / b 2 \pi r .
$$

Inserting equations (A2) and (A3) into (A4) and solving for $L$ yields

$$
L=\rho_{q} \sqrt{\frac{2 \pi r k}{b \tau_{q}}} .
$$

Inserting $\tau_{q}=\tau_{y}$ yields the critical distance $L_{\mathrm{c}}$ above which the electrostatic repulsive force does not induce glide between two isolated loops, if their elastic interaction is neglected. Taking a value of $\tau_{y}=0.5 \mathrm{MPa}, r=1 \mu \mathrm{m}$, equation (A5) yields a value for $L_{\mathrm{c}}$ equal to $1.7 \mu \mathrm{m}$ for the upper bound value of $10^{-10} \mathrm{C} / \mathrm{m}$ estimated above. This is to be compared to the critical equilibrium distance of $1-2 \mu \mathrm{m}$ between two uncharged loops. It thus seems that electrostatic interactions might have a measurable effect on the equilibrium position of closely spaced loops, if their line density is at equilibrium with the defect population in the crystal.

The above estimation is clearly an upper bound value since it was assumed that each loop carries an equilibrium charge. In our experiments however, the loops are punched and glide rapidly to their equilibrium position, typically in the $100 \mathrm{~s}$ necessary to cool the samples. It is therefore possible that the first few punched loops sweep out the defects as they move on their glide cylinder, resulting in charging. Since all the loops of the train glide on the same cylinder, they encounter fewer and fewer defects as the number of their predecessors increases. In the time considered, the typical diffusion distance as estimated by $(\mathrm{Dt})^{1 / 2}$ is in the order of $60 \mathrm{~nm}$. The total number of defects on a glide cylinder of length $20 \mu \mathrm{m}$, radius $1 \mu \mathrm{m}$ and "wall thickness" $60 \mathrm{~nm}$ at $300 \mathrm{~K}$ is thus about 1000 . If all these defects are swept by the first loop gliding on the cylinder, they induce a line charge on the order of $10^{-11} \mathrm{C} / \mathrm{m}$, depleting completely the diffusion volume and thus preventing the charging of the subsequent loops. If the defects are equally incorporated by the 100 loops of a train of that length, the line charge is 100 times less and has a negligible effect on the equilibrium spacing of the loops [equation (A5)].

It can be concluded from the semi-quantitative argument presented above that a row of loops which is at thermal equilibrium with the lattice before it glides might be influenced by electrostatic forces if the loops break away from their atmosphere of defects of opposite sign. On the other hand, if the loops move immediately after they have been nucleated, as is the case in our experiments, the diffusion rate is not high enough to charge them significantly and the electrostatic forces will be insignificant compared to the elastic repulsion between the loops. At the most, the first few loops of the train might become charged and their spacing might be larger than that predicted by elastic interactions only. 